\title{
Configurational Approach to Identify Concept Networks in selected Clinical Safety Incident Classes
}

\author{
Jaiprakash Gupta \\ University of Sydney \\ guptajs1@gmail.com
}

\author{
Simon Poon \\ University of Sydney \\ simon.poon@sydney.com.au
}

\begin{abstract}
Classifying clinical safety incidents (CSI) in their correct classes depends on the multiple concepts used to describe them. Machine learning based classification case study presented in this paper shows that it fails to identify the underlying complex concepts associations between the CSI classes. Two pairs of classes, each having high and low confused classes (as determined by the classifier), were further investigated by applying the set-theoretic-based logical synthesis methodology. The aim is to identify the relationships between concept networks for selected classes. The concept networks were identified using a set of 117 terms and measures taken included degree-centrality and in-betweenness centrality. In this study, using deterministic configurational approach, it is feasible to draw a meaningful relationship between concepts using the complex medical dataset sourced from the Incident Information Management System. The study is proof of concept that it is possible to identify concept networks and concept configuration rules for CSI classes.
\end{abstract}

\section{Introduction}

Improving safety and quality in a health care system depends on identifying, reporting, analyzing, learning and preventing things go wrong when treating patients. In pursuit of automating this process, the standard machine learning approaches (ML) focus on performances achieved on measures including accuracy and precision, and confusion matrix output to identify high and low performing class. Clinical safety incidents (CSI) are text documents and narratives about what caused or could have caused harm to the patient in the process of receiving care in the health sector. For illustration, a CSI report stating, "Patient received vancomycin tablets instead of prescribed 24 hours vancomycin infusion" is an example of medication class CSI. Automated real-time classification of CSI from clinical notes or incident reports has the potential to improve patient safety. Detecting patient harms occurring in hospitals and implementing strategies to prevent them [1] improves clinical governance in the hospital [2]. The magnitude of the incidence rate is alarming. On average between $5-10 \%$ for patients admitted to hospital [3] and 4\% for patients visiting health services in primary care [4] have one or more CSI. Manual coding of CSI from free-text documents to account for incident rates are expensive and inefficient. Hospitals now use commercially available software like the Incident Information Management System (IIMS) or other similar products for clinicians to record the CSI. To enter a report takes between 2060 minutes. An automated classification and reporting system that is accurate and consistent is highly desirable [5].

Classifying CSI reports in the correct class is a complicated task. Often the concepts used between the classes overlap, leading both human and machine alike to misclassify them. AI tools are highly sought after to solve this problem. The approaches taken to seek a solution for classifying CSI using AI have been limited to machine learning. The architecture of the Incident Information Management System (IIMS) used in Australia is very complicated, but at its core lies the Generic Reference Model (GRM) [6]. All the twelve amenable CSI classes using GRM taxonomy have not received performance evaluation and undergone automated classification [7]. Area of automated classification has been very contextual, Ong et al. [8] used binary models for investigating clinical handover, patient identification (2 sub-categories of clinical management class in GRM) and Wang et al. [9] added one more sub-category, deteriorating patient from the same class) and falls, medications, pressure injury, aggression, documentation, blood product, patient identification and infection types of clinical classes.

For a clinician choosing the right class for a CSI is a complicated task. To select right class information from multiple sources interact including, the environment, the context, the notifiers profession, the first language of the person notifying and the clinical experience they have, the choices of concepts available 
to them, the information gathered and the time to reflect.

In its simple form, it can be expressed using causality paradigm or simple logic if the incident has $\mathrm{X}_{1}, \ldots, \mathrm{X}_{\mathrm{n}}$ concepts then it would fall under class $\mathrm{Y}$, e.g. if the incident described has concepts including infection, MRSA, antibiotics, fever, sepsis, it would be classed as HAI class of incident.

Text classification approach (TC) segues well into a configurational approach as a form of logical synthesis is central to the latter. In TC, the bag-ofwords approach constrains the algorithm to use a specified number of concepts to train the classifier. In WEKA maximum of 999 concepts choice is provided. In the configurational approach, a specific combination of concepts (elements) determines to identify a specified class (outcome) [10]. The combination of elements in mathematics can be transposed as variables or attributes, or actors in sociology, and as nodes or vertices to form relations with links or edges in network analysis.

Several studies [11],[12],[13],[24],[28] have preferred a configurational approach, which rooted in set theory, over traditional correlational or multiregression methods in solving the high dimensional problem. The shift was primarily because conjunctural causation (i.e., multiples variables lead to an outcome), causal asymmetry (i.e., causal relationship is asymmetrical) and phenomenon of equifinality (i.e., different combination can lead to the same outcomes) were poorly addressed by correlation-based causality analysis [11],[12],[13]. Several concepts used in a CSI class description could occur in another CSI. Out study overcomes these limitations using configurational approach.

We first undertake the classification task to validate CSI reports classes automatically. For the first time, the classifier multinomial naïve Bayes is trained on data using a more efficient and improved World Health Organization patient safety classification (WHO) taxonomy. The overall and class-level performance achieved by the classifier and the confusion matrix outputs are used to direct the next classes and concept boundaries. Following this, a further investigation applying the set-theoretic-based logical synthesis methodology on two pairs of classes, each having high and low confused classes (as determined by the classifier) is undertaken to seek deeper level of understanding of concept boundaries, concept networks and concept cluster rules. Application of configurational analysis is novel to clinical incident classification and AI.

\section{Background}

Table 1 presents a brief outline of significant work in the configurational analysis space. Qualitative Comparative Analysis (QCA) has been well tested in sociology [10] other physical, behavioral, social, and biological sciences [23]. At its core, QCA is based on ideas from the field of logic synthesis to obtain the minimal Boolean sum-of-products (SOP) formulas that are adequately represented in a given truth table as variables. The truth table lists all logically possible combinations of the variables based on the dataset included in the study. QMA [22] is used for minimization of Boolean logic formulas to find the smallest, logically valid combination of variables that have the broadest coverage overall cases under investigation. The minimization process is based on repeatedly applying three laws of logic: 1) absorption, 2) idempotency or redundancy and 3) the law of excluded middle.

Table 1 Evolution of configurational analysis approach.

\begin{tabular}{|c|c|}
\hline Date & Authors and their work \\
\hline $\begin{array}{l}\text { Around } \\
40 \mathrm{BC}\end{array}$ & $\begin{array}{l}\text { Aristotle Organon - a collection of his six } \\
\text { works on logic [14] }\end{array}$ \\
\hline $\begin{array}{l}1847 \\
1854\end{array}$ & $\begin{array}{l}\text { Boole G. The Mathematical Analysis of } \\
\text { Logic. An investigation of the laws of } \\
\text { thought and mathematical theories of logic } \\
\text { and probabilities [15] }\end{array}$ \\
\hline 1880 & $\begin{array}{l}\text { Pierce C S. A Boolean Algebra with one } \\
\text { constant [16] }\end{array}$ \\
\hline 1880 & $\begin{array}{l}\text { Venn J. On the diagrammatic and } \\
\text { mechanical representation of propositions } \\
\text { and reasoning [17] }\end{array}$ \\
\hline 1922 & $\begin{array}{lll}\text { Wittgenstein L. } & \text { Tractatus } & \text { Logic- } \\
\text { Philosophicus [18] } & & \end{array}$ \\
\hline 1930 & $\begin{array}{l}\text { Shanon C.E. A Mathematical Theory of } \\
\text { Communication [19] }\end{array}$ \\
\hline 1953 & $\begin{array}{l}\text { Karnaugh M The map method for synthesis } \\
\text { of combinational logic circuits [20] }\end{array}$ \\
\hline 1955 & $\begin{array}{l}\text { Quine W.V.M A Way to Simplify Truth } \\
\text { Functions [21] }\end{array}$ \\
\hline 1956 & $\begin{array}{l}\text { McCluskey E Jr. Edward J.Minimization of } \\
\text { Boolean Functions [22] }\end{array}$ \\
\hline 1987 & $\begin{array}{l}\text { Ragin C.C The Comparative Method: } \\
\text { Moving Beyond Qualitative and } \\
\text { Quantitative Strategies.[11] }\end{array}$ \\
\hline 2003 & $\begin{array}{l}\text { Fišer F and Hlavička J "BOOM - A heuristic } \\
\text { Boolean minimizer [12] }\end{array}$ \\
\hline 2013 & $\begin{array}{l}\text { Su K.A. A novel approach to large scale } \\
\text { casual complexity analysis - CANAL [24] }\end{array}$ \\
\hline 2014 & $\begin{array}{l}\text { H Su. Introducing a Social-Enabled } \\
\text { Deterministic Model for Causality } \\
\text { Reasoning - CARE. [24] }\end{array}$ \\
\hline
\end{tabular}


A logical analysis method, like (QMA), play the role of analyst and lay a strong foundation for analysis. After listing all variables in a truth table, the analyst needs to select the threshold at which sufficient evidence for the outcome is defined.

Overtime with an increase in several causal variables limited the use of traditional QCA tools as the processing time increased exponentially with every additional variable. An exact analysis of 35 variables and one outcome requires 1.8 Petabytes of memory [24],[25]. To overcome this limitation of processing time issues, new processing frameworks, including BOOM [12], and CARE [24] developed.

Early approaches using Boolean minimization method primary focused on obtaining a minimal solution to extract causal pathways (as configurations). The introduction of the BOOM was to handle higher dimension datasets and look at all the causal variables as if they were independent of each other. The computation efficiency gained was mainly due to the non-deterministic nature of the BOOM algorithm. Procedurally, individual factors ranked and selected as implicant based on its impact on the outcome. When there are two or more factors with a similar impact on the outcome, one factor is selected randomly as implicant.

Move toward causality reasoning from causality analysis and from heuristic to deterministic solutions lead to the development CARE [24]. In CARE [24], the interactions between the causal variable were considered in the solution. Also, need to incorporate the interactions of the variables using (measures like centrality) was develop to arrive at more meaningful configurations, i.e., not treating variables independent, and also making the algorithm more deterministic

In Table 2, the key differences between various causality processing frameworks are shown, for more information on these frameworks, readers are directed to read works of $\mathrm{Su}[24],[26]$.

Table 2 Key differences between causality processing frameworks

\begin{tabular}{|c|c|c|c|c|c|l|}
\hline $\mathrm{B}$ & $\mathrm{K}$ & $\mathrm{Q}$ & $\mathrm{Q}$ & $\mathrm{B}$ & $\mathrm{C}$ & Key \\
$\mathrm{A}$ & $\mathrm{M}$ & $\mathrm{M}$ & $\mathrm{C}$ & $\mathrm{O}$ & $\mathrm{A}$ & features \\
{$[15]$} & $\mathrm{A}$ & $\mathrm{A}$ & $\mathrm{A}$ & $\mathrm{O}$ & $\mathrm{R}$ & \\
\hline & $\mathrm{P}$ & {$[21]$} & {$[11]$} & $\begin{array}{c}\mathrm{M} \\
\mathrm{E}\end{array}$ & $\begin{array}{c}\mathrm{E} \\
{[24]}\end{array}$ & \\
\hline$<3$ & $<4$ & $<6$ & $<50$ & $>50$ & $>50$ & $\begin{array}{l}\text { Good for N } \\
\text { variables }\end{array}$ \\
\hline 1 & 1 & 2 & 2 & 3 & 3 & $\begin{array}{l}\text { Staged } \\
\text { process }\end{array}$ \\
\hline & & $\checkmark$ & $\checkmark$ & $\checkmark$ & $\checkmark$ & $\begin{array}{l}\text { Finds prime } \\
\text { implicant } \\
\text { (PI) }\end{array}$ \\
\hline & & $\checkmark$ & $\checkmark$ & $\checkmark$ & $\checkmark$ & $\begin{array}{l}\text { Finds } \\
\text { Essential PI } \\
\text { (EPI) }\end{array}$ \\
\hline
\end{tabular}

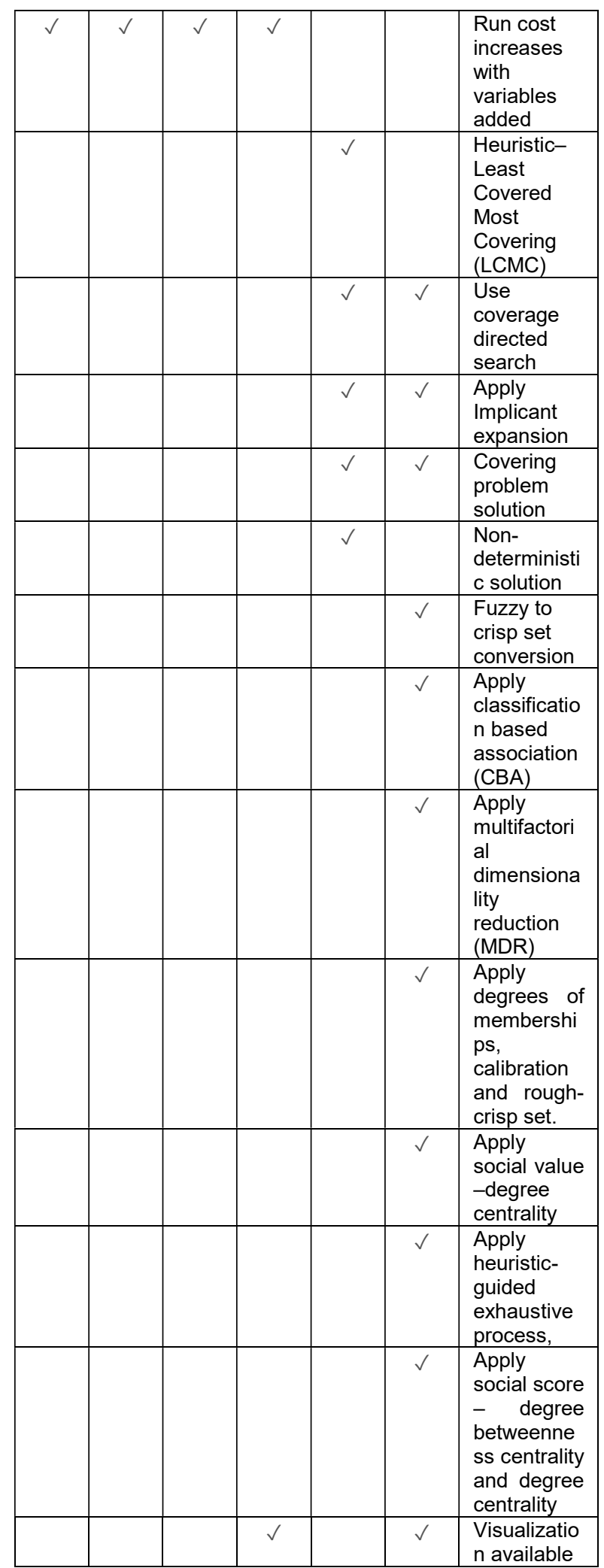

Using this Problem statement: A combination of concepts A, B or a combination of concept $\mathrm{C}$ and $\mathrm{D}$ 
lead to class outcome $\mathrm{Z}$. In Boolean notation, this is represented as $A^{*} B+C^{*} D \rightarrow Z$. It is necessary then to first undergo a minimization procedure that identifies the most straightforward set of concepts that can account all the observed class, as well as their absence using Boolean algebra. In TC analysis, it is common to consider hundreds of concepts, in our case, top 300600 concepts with high information gain are adequate for the classifier to perform optimally, albeit the use of CARE was also suited. We also know that some classes had high accuracy than others. Thus, a matrix using rows for the incident, columns for concepts marked as a present $(+)$ or absent $(-)$ in a class and the rightmost column representing the class under investigation as $(+)$ for that class and remain class incidents as negative, thus establishing a truth table. QCA approach is driven by theory with the preset notion in our case that the cluster of concepts for a class is in the selected concepts. The coding of the presence/absence of concepts also requires a clear view of that concept and when and where it can be considered present. The dichotomization of quantitative measures about the classes also needs to be carried out with an explicit rationale. The determination of concept cluster represented in the sets may also be revised in the light of the results of the analysis if some configurations are still shown as being associated with other classes.

\section{Method}

The data from seven hospitals in one state in Australia that used IIMS program to record CSIs were used for this study. After seeking approval from ethics governing bodies of these hospitals, the data between January 2004 and December 2012, was downloaded. The CSI reports were de-identified by a person (patients and health staff names in the reports) and facility information as per the ethics approval conditions. The data fields selected from IIMS included service type, title, incident description, contributing factors, initial actions taken and outcomes of the incident.

In IIMS, each clinical incident is an independent document of the instance class. For the classification experiment, 3600 CSI reports (300 reports for each of the 12 categories) were used. The 12 natural CSI classes applying Generic Reference Model in IIMS include; Aggression Aggressor (AA), Aggression Victim (AV), Blood and Blood Product (BBP), Behavior and Human Performance (BHP), Clinical Management (CM), Documentation (DOC), Fall (FALL), Hospital Associated Infection/infestation (HAI), Medication (MED), Nutrition (NUT),
Pathology Lab (PATH) and Pressure Ulcers (PU). The brief description of these classes is available [17]. These classes were chosen for the reasons as they occur more frequently, are reported often, are repeatedly investigated, carry a high risk to the patient and hospital, and they were amenable for investigation.

An expert-1 (JG) relabeled the 3600 clinicians labelled CSI reports applying classes label using IWHO taxonomy (Table-3). After that, another expert-2 relabeled $50 \%$ (1800 CSIs) of the data applying IWHO taxonomy. Both the experts are senior clinicians and managers together having over 30 years of experience using IIMS. The Fleiss' Kappa [7] statistics were used to measure inter-labelling reliability expert1 and expert-2. The inter-expert reliability between expert-1 and expert-2 achieved a Fleiss' Kappa of 0.98 (CI $0.98-0.99$ ). Six reports with insufficient information to designate them a class were placed in an unlabeled class (UC) folder.

Table 3 Distribution of clinical safety incidents (CSI) classes applying

\begin{tabular}{|c|c|c|}
\hline $\begin{array}{l}\text { Taxonomies } \\
\text { GRM }\end{array}$ & WHO_I & \\
\hline Classes ( $\mathrm{N}=300$ per class) & Classes & $\mathrm{N}$ \\
\hline Aggression Aggressor (AA) & \multirow{2}{*}{$\mathrm{BEH}$} & \multirow{2}{*}{848} \\
\hline Aggression Victim (AV) & & \\
\hline Blood and Blood Product (BBP) & BBP & 271 \\
\hline Pathology Lab (PATH) & PATH & 401 \\
\hline $\begin{array}{l}\text { Behaviour \& Human } \\
\text { Performance (BHP) }\end{array}$ & \multirow[t]{2}{*}{ CLP } & \multirow[t]{2}{*}{327} \\
\hline Clinical Management (CM) & & \\
\hline Documentation (DOC) & DOC & 206 \\
\hline Falls (FALL) & PTA & 325 \\
\hline $\begin{array}{l}\text { Hospital Associated Infection } \\
\text { (HAl) }\end{array}$ & $\mathrm{HAl}$ & 298 \\
\hline Medication (MED) & MED & 324 \\
\hline Nutrition (NUT) & NUT & 300 \\
\hline Pressure Ulcers (PU) & $\mathrm{PU}$ & 294 \\
\hline Unclassified Class (UC) & $\mathrm{UC}$ & 6 \\
\hline CSIs Total & & 3600 \\
\hline
\end{tabular}

The classification performance analysis was undertaken using the Waikato Environment for Knowledge Analysis ${ }^{7}$ (WEKA) open source software. The data was trained on multinomial naïve Bayes classifier. Advanced feature selection strategies applied on the classifier included; using evaluator information gain (IG), search method ranker and processing of 600 concepts. The algorithm randomly divides the classifier into ten partitions, and nine parts were used as a training set and one part as the test set and then iterating the whole process ten times. This was done to reduce sampling bias and obtain the best estimate of 
the performance of the classifiers. The performance measures applied in this study are overall accuracy $(\mathrm{ACU})$, precision (PRE) and $\mathrm{F}$ measure $(\mathrm{FM})$ and Area Under the Curve (AUC) measure.

The 3600 CSI had 297,553 concepts and 11,708 unique concepts. For configurational analysis CARE software, which is developed [23] and published [26] was applied. The CSV data file prepared for MNB classifier was prepared for CARE software. The class output column was transformed using 1 as on-set value for the CSI class under investigation and 0 value as off-set for the remaining CSI. To reduce the dimensionality of the dataset from 600 concepts to manageable size. During the pre-processing stage concepts in the solutions for each of the class were collated disregarding the literal variable forms (symbolized as " * " asterisk for AND and " " Tilda for Not used Don't care). The duplicate concepts were removed, and a final set of 117 unique concepts formed the truth table. The 3600 CSI formed the rows and 117 concepts the column. The presence of the concepts is indicated by the value " 1 ", if the concept was present more than once in it was flatten to 1 . The absence of the concept and indicated as " 0 ". The last column indicated the output as onset (' 1 ') or offset (' 0 ') depending on the class analyzed. The matrix had 421,299 cells, with 23,554 indicating " 1 " and rest had " 0 " values. In this paper, 4 classes were selected for configurational analysis, and they were PU, NUT, DOC and CLP. They were selected based on accuracy achieved on the MNB classier, classes PU and NUT had high accuracy achieved, and CLP and DOC with low accuracy achieved. Table 4 summarizes the number of 1's, representing AND (*), 0's representing NO $(\sim)$ and DON'T CARE (DC) concepts, a number of on-set and off-set cases of each of the CSI classes investigated.

Table 4 Number of concepts in clinical safety incidents (CSI) classes pre-processed for configurational analysis.

\begin{tabular}{ccccc}
\hline Classes & N On-set & N Off-Set & 1's & 0's \\
\hline PU & 294 & 3306 & 2552 & 31,963 \\
\hline NUT & 300 & 3300 & 2089 & 33,011 \\
\hline CLP & 327 & 3273 & 1668 & 36,591 \\
\hline DOC & 206 & 3394 & 1305 & 22,797 \\
\hline
\end{tabular}

Four processes are undertaken using CARE software [24] included; a) coverage directed search (CDS), b) Implicant expansion (IE), c) solution coverage (SC) and d) concept network. The measures reported for CDS are processing time for the program, number of implicants, number of positive values and average raw coverage (RC). The measures reported for IE are processing time for the program, number of implicants, number of positive values and average raw coverage (RC). The measures reported for SC are processing time for the program, number of solutions, number of positive values and solution coverage (SC). The solutions rules exemplars, the shortest and longest solution rule for each class are presented. The concepts in the solution were aggregated, and 4 lists were developed; a) list of concept unique concepts appearing once only, those appearing twice, thrice and in all 4 classes. In this list, frequency concepts identified as AND and NO or DC, along with the degree of centrality and degree betweenness score are presented. For visualization first, a graph with all the causal concepts correlation and a mixed graph showing correlation and solutions are presented. The correlations graph shows nodes with concept name and the size of the node indicating the positive literal frequency, the links between the nodes show positive, negative and degree of correlations by a line, dotted line and thickness of the line respectively. The mixed graph shows the network of concepts in the solution (red color dots) and those that are not part of the solution (blue dots). The size of the red nodes indicates the literal coverage with unique or participation as NO or DC; the thickness of the link between nodes reflect their frequency.

\section{Results}

Table 5 summarizes the overall performance achieved by classifier MNB arranged by best to the poor performance by classes. The overall accuracy (ACU) achieved by the classifier is $85 \%$ and class pressure ulcer (PU) achieving 98\% the highest ACU with the classes and reciprocal FM score of $84 \%$ and $94 \%$ were achieved respectively. The overall precision (PRE) achieved by the classifier is $83 \%$ with class NUT achieving $96 \%$. The lowest ACU was achieved by classes documentation DOC (36\%) and clinical processing CLP (66\%).

Table 6 shows the confusion matrix output with an actual number of CSI classes in rows and the predicted the number of CSI classes in the column. The classes DOC and CLP shows spread of CSI over other classes indicative of confusion as compared to classes PU or NUT. In Table 7 results of running the CDS component of the CARE program, shows that the run time for classes PU and NUT is comparatively shorter than that for classes CLP and DOC. The number of implicants and average raw coverage (RC) show similar variation in these two class clusters.

Table 5 Overall and class level accuracy (ACU), precision (PRE), f-measure (FM) and area under the 
curve (AUC) achieved by classifiers multinomial naïve Bayes (MNB) on Improved World Health Organization (WHO-I) taxonomy for clinical safety incident classification.

\begin{tabular}{|c|c|c|c|c|c|}
\hline MNB & N & ACU & PRE & FM & AUC \\
\hline PU & 294 & 0.98 & 0.91 & 0.94 & 1.00 \\
\hline BEH & 848 & 0.95 & 0.87 & 0.91 & 0.99 \\
\hline PTA & 325 & 0.93 & 0.94 & 0.93 & 0.99 \\
\hline HAI & 298 & 0.92 & 0.87 & 0.89 & 0.99 \\
\hline NUT & 300 & 0.90 & 0.96 & 0.93 & 0.99 \\
\hline BBP & 271 & 0.87 & 0.80 & 0.83 & 0.98 \\
\hline MED & 324 & 0.85 & 0.82 & 0.84 & 0.98 \\
\hline PATH & 401 & 0.84 & 0.80 & 0.82 & 0.98 \\
\hline CLP & 327 & 0.66 & 0.67 & 0.67 & 0.94 \\
\hline DOC & 206 & 0.36 & 0.62 & 0.45 & 0.92 \\
\hline UC & 6 & 0.00 & 0.00 & 0.00 & 0.92 \\
\hline Overall & 3600 & 0.85 & 0.83 & 0.84 & 0.97 \\
\hline
\end{tabular}

In the CDS run for class PU there were 102 implicant clusters and a total of 324 positive implicants identified. Though the average RC was 0.011 the highest RC of 0.087 for one implant cluster; "applied*, area*, care*, dressing*, pressure*" occurred 25 times. In the run for class NUT, highest RC of 0.466 for "diet*, fluid*" was noted and 136 positive implicants were identified. In class CLP implicants "birth*, $\sim$ home*, iv $\sim *$, $\sim$ notified $*, \sim$ poor $* \sim$ sent $*, \sim$ specified *, ward" had highest RC of 0.085 and 25 positive $\mathrm{N}$. For DOC class Implicants; " blood *, correct*, emergency* $\sim$ error*, form*, $\sim$ group $*$ labelled $*$, laboratory*, $\sim$ medicine*, $\sim$ notified, pathology*, received*, $\sim$ recollection*, request*, specimen*" had RC 0.029 and 5 positive $\mathrm{N}$. The lowest RC 0.003 implicants; 'aged*, applied*, area*, care $* \sim$ dressing*, $\sim$ pressure*, $\sim$ sacrum with 1 positive $\mathrm{N}$ for class PU.

Table 6 Confusion matrix outcomes are applying WHO-I taxonomy.

\begin{tabular}{lccccccccccc}
\hline Class & PATH & DOC & CLP & HAI & MED & BBP & NUT & PTA & BEH & PU & UC \\
\hline PATH & 338 & 36 & 5 & 3 & 0 & 18 & 1 & 0 & 0 & 0 & 0 \\
DOC & 57 & 74 & 28 & 1 & 14 & 29 & 0 & 2 & 0 & 1 & 0 \\
CLP & 10 & 4 & 216 & 23 & 19 & 6 & 7 & 16 & 13 & 13 & 0 \\
HAI & 1 & 0 & 8 & 274 & 1 & 1 & 0 & 4 & 1 & 8 & 0 \\
MED & 1 & 0 & 22 & 9 & 275 & 3 & 2 & 11 & 0 & 0 & 1 \\
BBP & 14 & 6 & 9 & 0 & 6 & 235 & 0 & 0 & 1 & 0 & 0 \\
NUT & 0 & 0 & 10 & 1 & 12 & 0 & 271 & 4 & 0 & 1 & 1 \\
PTA & 1 & 0 & 20 & 2 & 7 & 1 & 0 & 785 & 29 & 3 & 0 \\
BEH & 0 & 0 & 2 & 0 & 0 & 0 & 1 & 10 & 308 & 3 & 1 \\
PU & 0 & 0 & 0 & 2 & 0 & 0 & 0 & 0 & 3 & 289 & 0 \\
UC & 1 & 0 & 3 & 0 & 0 & 0 & 0 & 1 & 1 & 0 & 0 \\
\hline
\end{tabular}

Table 7 Clinical safety incidents classes CDS results

\begin{tabular}{ccccc}
\hline Classes & $\begin{array}{c}\text { Run time } \\
(\mathrm{sec})\end{array}$ & $\begin{array}{c}\mathrm{N} \\
\text { Implicants }\end{array}$ & $\begin{array}{c}\text { Positive } \\
\mathrm{N}\end{array}$ & $\begin{array}{c}\text { Mean } \\
\text { RC }\end{array}$ \\
\hline PU & 2.633 & 102 & 324 & 0.011 \\
\hline NUT & 13.976 & 87 & 311 & 0.012 \\
\hline CLP & 39.383 & 225 & 590 & 0.009 \\
\hline DOC & 31.738 & 125 & 175 & 0.008 \\
\hline
\end{tabular}

In Table 8 results of running the implicant expansion component of CARE program, shows that the run time for all the classes was much shorter than that of CDS. The number of implicant cluster reduced, and with an increase in positive literals, the average raw coverage also improved for every class. In the IE run for class PU, there were 76 implicant clusters and a total of 1854 positive implicants identified. Though the average $\mathrm{RC}$ was 0.085 the highest $\mathrm{RC}$ of 0.222 for one implant cluster;
Table 8 Clinical safety incidents classes Implicant Expansion results

\begin{tabular}{ccccc}
\hline Classes & $\begin{array}{c}\text { Run time } \\
\text { (sec.) }\end{array}$ & $\begin{array}{c}\mathrm{N} \\
\text { Implicant }\end{array}$ & $\begin{array}{c}\text { Positive } \\
\mathrm{N}\end{array}$ & $\begin{array}{c}\text { Mean } \\
\mathrm{RC}\end{array}$ \\
\hline PU & 2.098 & 76 & 1854 & 0.085 \\
\hline NUT & 0.801 & 75 & 1465 & 0.067 \\
\hline CLP & 3.893 & 218 & 573 & 0.012 \\
\hline DOC & 0.096 & 120 & 279 & 0.014 \\
\hline
\end{tabular}

"pressure * $\sim$ skin $*$ ulcer*" occurring 65 times. In the run for class NUT, highest RC of 0.613 for " $\sim$ blood* diet* obstetrics *poor"' was noted and 176 positive implicants were identified. In class CLP implicants "birth*, $\sim$ home*, $\sim$ iv*, $\sim$ notified $* \sim$ poor $* \sim$ sent $*, \sim$ specified *, $\sim$ ward" had highest RC of 0.085 and 25 positive N. For DOC class Implicants; " medicine* $\sim$ notified $^{*}$ received* $\sim$ request * unlabeled*, $\sim$ ward" had RC 0.064 and 11 positive $\mathrm{N}$. The lowest RC 0.003 implicants; bed $*$ dressing $*$ given $*$ head $*$ 
injury * left * tear, with 1 positive $\mathrm{N}$ for class PU was noted.

The summary of the final stage of CARE program output on SC are presented in Table 9. The run time for the classes further improves over IE stage analysis. The number of solutions shows that classes PU and NUT had solution coverage of 1 with 41 and 48 solution rules each. On the other hand, classes, DOC and CLP solution coverage was under 1 with 87 and 153 solution rules, but still, complete solution coverage wants not achieved.

Table 9 Clinical safety incidents classes solution coverage results

\begin{tabular}{ccccc}
\hline Classes & $\begin{array}{c}\text { Run time } \\
(\mathrm{sec})\end{array}$ & Solutions & $\begin{array}{c}\text { Positive } \\
\mathrm{N}\end{array}$ & Sol.Cov \\
\hline PU & 0.147 & 41 & 288 & 1 \\
\hline NUT & 0.801 & 48 & 292 & 1 \\
\hline CLP & 2.563 & 153 & 295 & $<1$ \\
\hline DOC & 0.096 & 87 & 170 & $<1$ \\
\hline
\end{tabular}

In Table 10 some of the shortest and longest string of concepts in the solutions are showcased, and on review, the concepts appear multiple times in the solutions in various literals forms (as * or $\sim$ ). From the 117 concepts investigated 42 appeared in class PU solution, 76 in CSI class NUT, 91 in CLP and 84 in DOC (Table 11). The breakdown of these concepts appearing as AND and $\sim$ in the solutions shows, e.g., in the class PU the 42 concepts appeared 193 times (as AND 98 times and $\sim 95$ times) in the solution rules. Similarly, in class CLP the 91 concepts from the total of 117, appeared 1250 time (as AND 393 times and $\sim 857$ times) in the solutions. The configurational approach taken shows, considering the four classes investigated, using 117 concepts, 114 were used by them in different combinations. Further analysis of solutions showed that of the 114 concepts in solution 20 appeared once, and the distribution by classes is as shown below:

- $\quad \mathrm{PU}\left(4^{*}\right.$ : air, blister, broken, sore),

- $\quad$ NUT (3: antibiotics, $\sim$ door , floor )

- $\quad$ CLP $\left(3^{*} 7 \sim\right.$ : assistance*, cleaned*, aggressive*, $\sim$ absconded, $\sim$ bedside, $\sim$ charge, $\sim$ feet, $\sim$ verbal, $\sim$ walk, aggressor) and

- $\operatorname{DOC}(1 * 2 \sim$ : laboratory*, $\sim$ bank, $\sim$ signature $)$ the concepts.

It was also found $24 / 114$ concepts appeared in two classes (e.g. concept "form" appeared as * within classes CLP and DOC their degree centrality was 0.031 and 0.882 and degree betweenness was 0 and 0.043 respectively; concept "cells" appeared as AND* in class DOC and as $\sim$ ' in class DOC, and concept "confusion" appeared as $\sim$ ' in classes DOC and NUT). Similarly, various combinations of 34 concepts appeared in 3 classes, e.g. concept "admission" as AND in class CLP and DOC in class NUT. The remaining 31 concepts appeared in all four classes, e.g. concept "fluid" was AND for classes DOC and NUT, and as $\sim$ ' in classes CLP and DOC.

Table 10 Clinical safety incidents classes identified shortest and long solutions exemplar

\begin{tabular}{|c|c|}
\hline Classes & Solution details \\
\hline PU & $\begin{array}{l}\text { a) } \\
\text { b) }{ }^{*} \text { pressure }{ }^{*} \sim \text { ward } \\
\sim \text { area }{ }^{*} \sim \text { attended }{ }^{*} \sim \text { broken }{ }^{*} \text { care } \\
\sim{ }^{*} \sim \text { injury }{ }^{*} \sim \text { iv }{ }^{*} \sim \text { order }{ }^{*} \sim \\
\text { pressure }{ }^{*} \sim \text { refused }{ }^{*} \sim \text { result } \sim \\
{ }^{*} \text { sent }{ }^{*} \text { skin }{ }^{*} \sim \text { specified }{ }^{*} \sim \text { state }{ }^{*} \sim \\
\text { tear }{ }^{*} \sim \text { ward }\end{array}$ \\
\hline NUT & 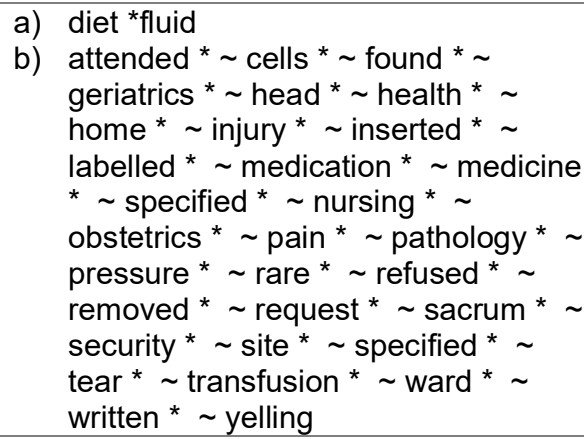 \\
\hline CLP & 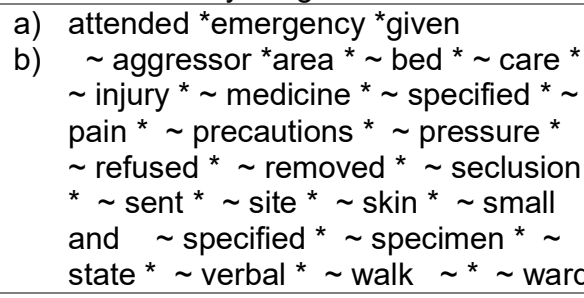 \\
\hline DOC & 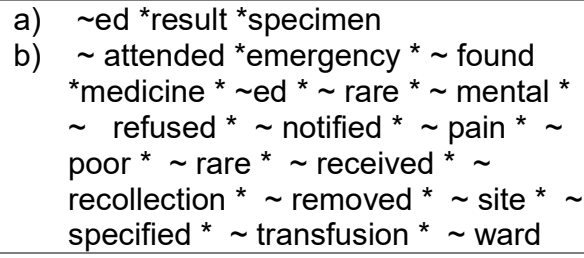 \\
\hline
\end{tabular}

A better way to understand the configuration of concepts in the classes is by using concept network graphs. In Figure 11 one all the 117 concepts are presented as nodes network, and the relationship is the correlation between them. The positive literal frequency of the labelled concept determines the node size. As seen concepts like care, blood, aged, received are more significant weight compared to nodes for, e.g. fell, soar, tear The line connecting the nodes shows if these concepts have:

- Positive correlation (lines), e.g., medicine - 
charted

- Negative correlation (dotted lines), e.g., incorrect - correct, searched, absconded

- Degree or strength of correlation (thickness of the line), e.g., aged - care, blood - form.

Not all concepts are correlated in the network graph; there are concepts like door, tube, blue, are standalone. It is clear that some concepts have tighter networks (lower right section of the graph) than the others (top left section of the graph).
Table 11 Clinical safety incidents classes Solution coverage concepts distribution $\left({ }^{*}=\right.$ AND, $\sim=$ Not sure/Don't care)

\begin{tabular}{|c|c|c|c|c|}
\hline Classes & Concepts & ${ }^{*}(1)$ & $\sim(0)$ & Total \\
\hline PU & $42\left(25^{*} \sim 17\right)$ & 98 & 95 & 193 \\
\hline NUT & $76\left(33^{*} \sim 43\right)$ & 199 & 452 & 651 \\
\hline CLP & $91\left(59^{*} \sim 32\right)$ & 393 & 857 & 1250 \\
\hline DOC & $84\left(51^{*} \sim 33\right)$ & 217 & 372 & 598 \\
\hline
\end{tabular}

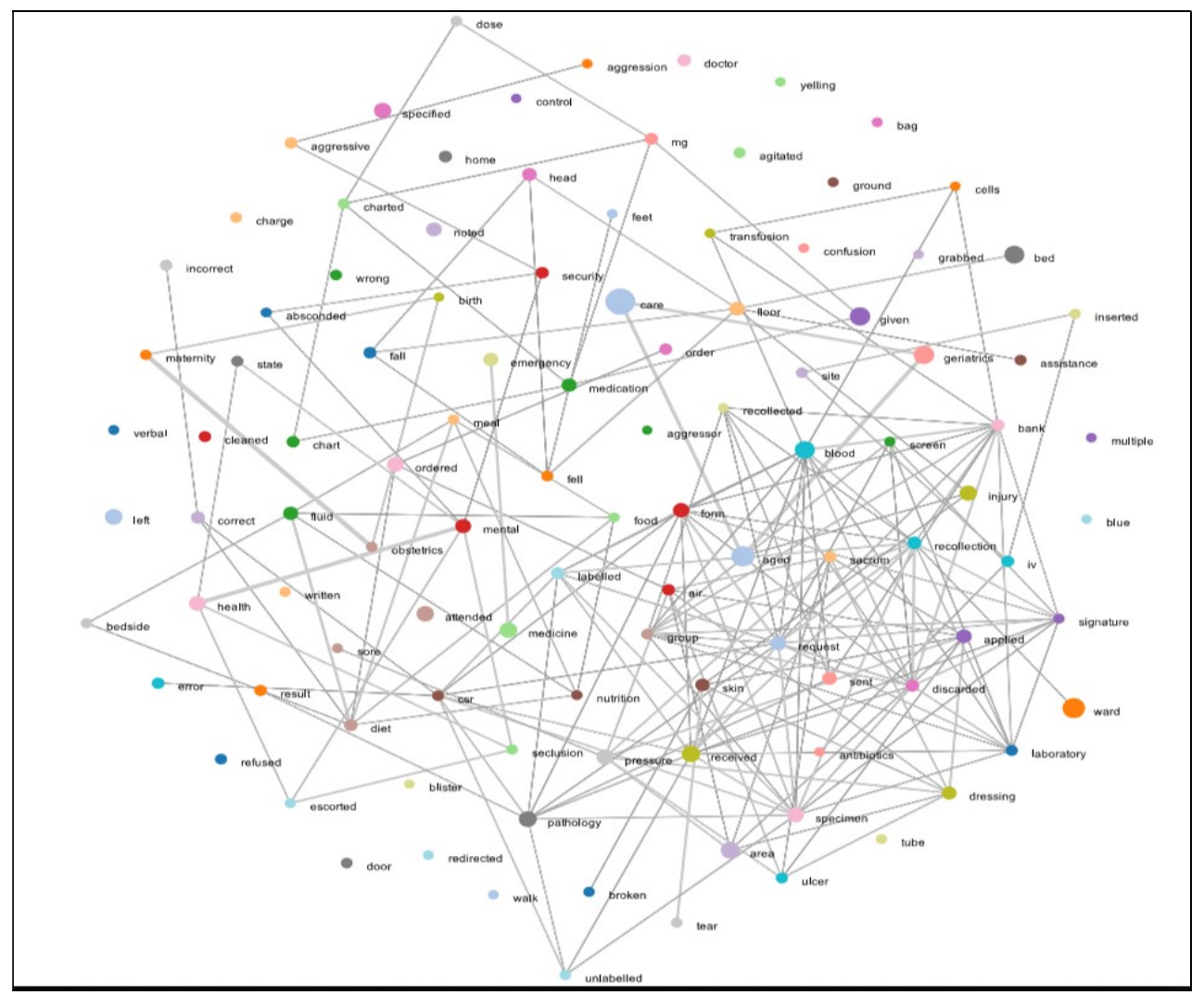

Figure 1 Concept Network graph for 117 concepts investigating 4 classes shows concept frequency (size of the node), positive correlation (lines), negative correlation (dotted lines), degree of correlation (thickness of the line) between the concepts and centrality on concepts

Figures 2-5 illustrates that each of the CSI classes has very distinct concept networks. The red nodes in these graphs are concepts in the solutions. The size of the red node reflects the concept participation frequency. The thickness of the links between the red nodes indicates their frequency of occurrences, and they cover all the solutions. The blue nodes represent the concepts that are not in the solution; the size reflects positive (AND concepts) occurrence frequency and lines linking the positive correlation between the concepts (if the lines are dotted it is indicative of negative correlation). Each concept network shows its role in the solutions. As noted in earlier SC section, classes PU and NUT had solution coverage of 1 , and this is reflected in the fig. 2 and 3 when compared to fig 4 and 5 showing concept network for classes DOC and CLP. 


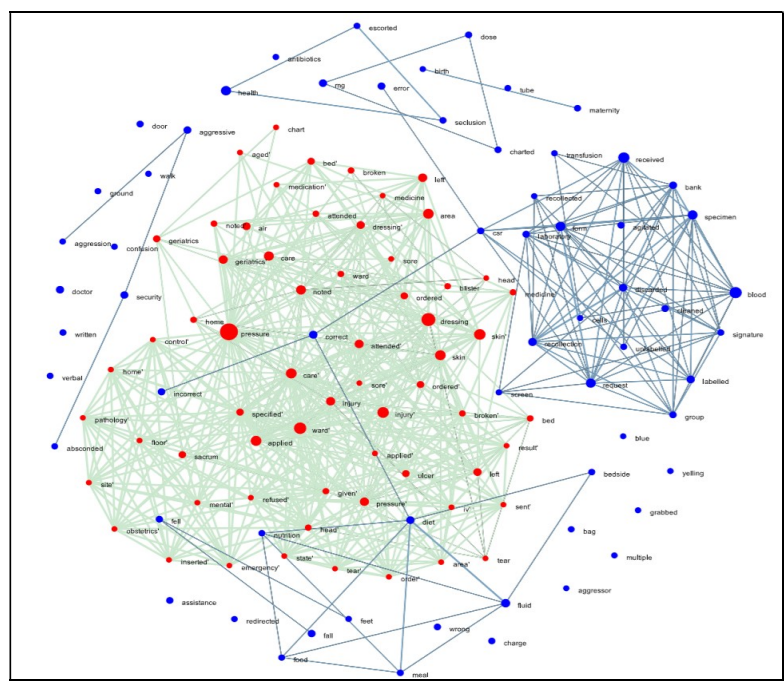

Figure 2 Mixed graph showing concept correlation and concepts in the solutions for clinical safety class pressure ulcer

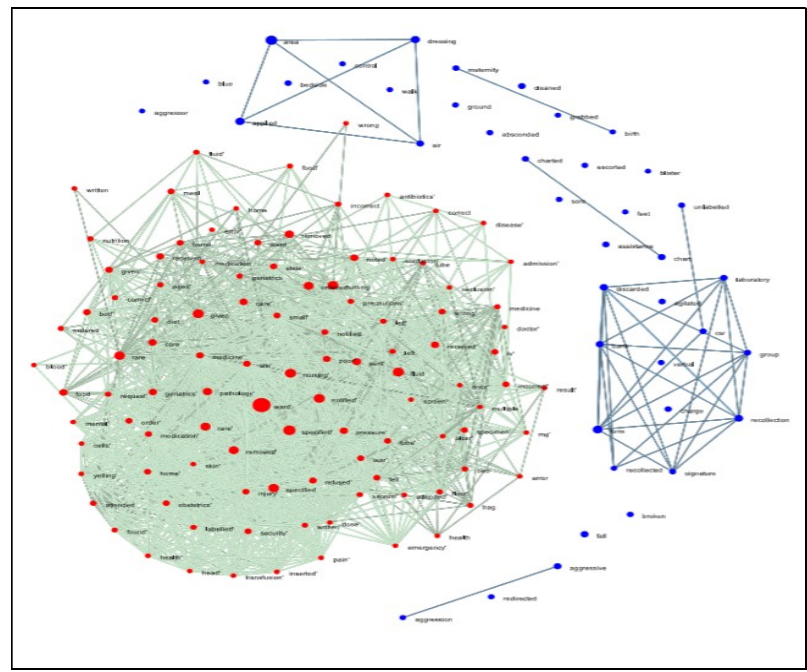

Figure 4 Mixed graph showing concept correlation and concepts in the solutions for clinical safety class nutrition.

\section{Conclusion}

The limitation of DM in resolving classification solutions for CSI taxonomy and the confusion matrix outputs are better understood using configurational approach. Application of configurational analysis is novel to clinical incident classification and AI. The set-theoretic based configurational approach is now sought after as it provides a window to see how concept clusters are interconnected and organized. The best part is the holistic and systemic approach that leaves no stone unturned. Though very few concepts are unique, those that appear multiple time

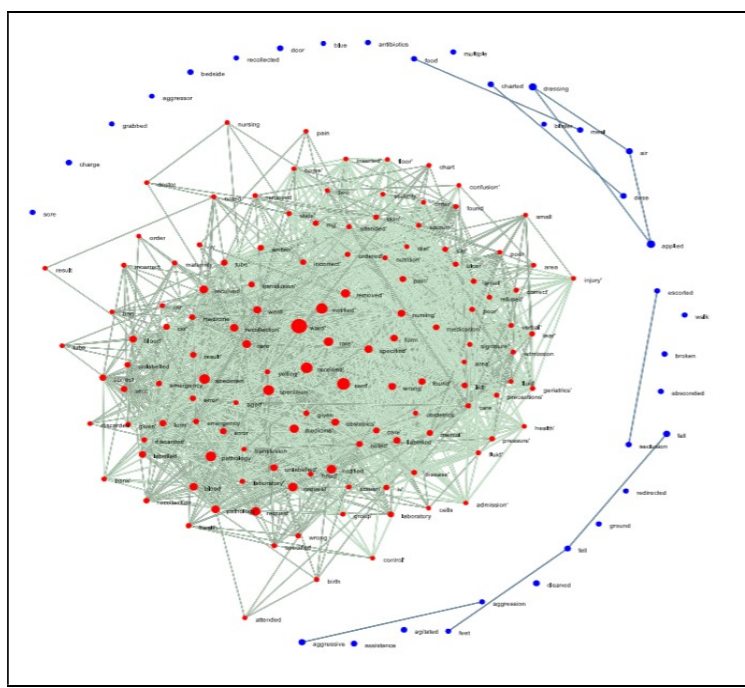

Figure 3 Mixed graph showing concept correlation and concepts in the solutions for clinical safety class documentation.

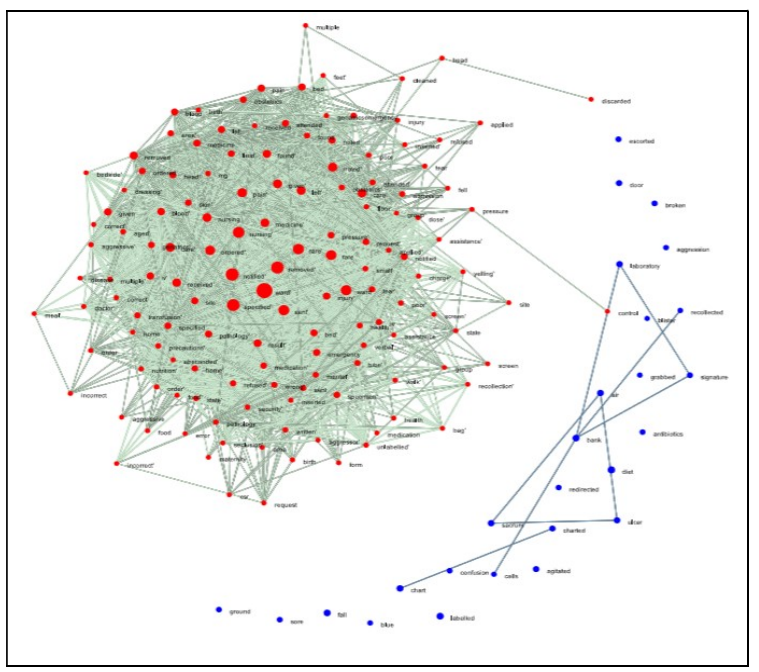

Figure 5 Mixed graph showing concept correlation and concepts in the solutions for clinical safety class clinical process

in different networks have different values of degree of centrality and betweenness, e.g. the concept "form" appeared as * in classes CLP and DOC their degree centrality was 0.031 and 0.882 and degree betweenness was 0 and 0.043 respectively. Thus, the concepts, even if they appear multiple time, they carry different weights and help in develop concept networks for each class.

The paper is proof of concept that complex and high dimensional dataset like CSI reports can be approached successfully with the deterministic configurational analysis framework. In AI for automation, these concept networks can assist in 
setting up concept boundaries and improve search engines performance.

\section{References}

[1] M. Govindan, A.D. Van Citters, E.C, Nelson, et al., Automated detection of harm in healthcare with information technology: a systematic review. BMJ Quality \& Safety .19 (2010), 1-11.

[2] D. Pittet, L. Donaldson. Challenging the world: patient safety and health care associated infection. International $\mathrm{J}$ Quality in Health Care. 18 (2006), 4-6.

[3] C. Vincent, G. Neale, M. Woloshynowych. Adverse events in British hospitals: preliminary retrospective record review. BMJ 2001; 322:517-19.

[4] S.S. Panesar, D. deSilva, A. Carson-Stevens A, et al. How safe is primary care? A systematic review BMJ Qual Saf 2016; 25:544-553.

[5] AHIMA. Computer assisted coding e-HIM workgroup. Delving into computer-assisted coding. J AHIMA. 2010; 75: $48 \mathrm{~A}-48 \mathrm{H}$

[6] J. Gupta, J. Patrick. Automated validation of patient safety clinical incident classification: macro analysis. Stud Health Technol Inform. 2013;188:52-7.

[7] J. Gupta, I. Korinska, J. Patrick. Automated validation of patient safety clinical incident classification. In: Georgiou et al. editors. Driving Reform: Digital Health is Everyone's Business. Open Acces: IOS Press; 2015.

[8] M. Ong, F. Magrabi, E. Coiera. Automated categorization of clinical incident reports using statistical text classification. Qual Saf Health Care 2010; 19: 1-7.

[9] Y. Wang, E. Coiera, W. Runciman, F. Magrabi. Using multiclass classification to automate the identification of patient safety incident reports by type and severity. BMC Medical Informatics and Decision Making, 2017; 17: 84.

[10] B. Rihoux, et.al., "From Niche to Mainstream Method? A Comprehensive Mapping of QCA Applications in Journal Articles from 1984 to 2011," Political Research Quarterly, pp. 175-184, 2013.

[11] C. C. Ragin, "The Comparative Method. Moving Beyond Qualitative and Quantitative Strategies," Berkeley: University of California Press, 1987.

[12] P. Fišer and J. Hlavička, "BOOM - A heuristic Boolean minimizer," Computing and Informatics, pp. 1951, 2003.

[13] P. C. Fiss, "A Set-Theoretic Approach to Organizational Configurations," The Academy of Management Review, vol. 32, pp. 1180-1198, 2007.

[14] R. Smith. "Aristotle's Logic", The Stanford Encyclopedia of Philosophy (Summer 2019 Edition), Edward N. Zalta (ed.) Cited 2019 May 30; Available: https://plato.stanford.edu/archives/sum2019/entries/aristotle $\underline{- \text { logic/. }}$

[15] G. Boole. An Investigation of the Laws of Thought. Prometheus Books. 2003

[16] A. S. Peirce. Collected Papers of Charles Sanders Peirce In: Exact Logic and The Simplest Mathematics. Hartshorne V and Weiss P (eds) Vol 3. Pg 13. Harvard University Press 1993.

[17] J. Venn. On the Diagrammatic and Mechanical Representation of Propositions and Reasoning. The London, Edinburgh, and Dublin Philosophical Magazine and Journal of Science. 1918. 5. 10 (59): 1-18.

[18] G. H. von Wright (1955). "Ludwig Wittgenstein, A Biographical Sketch". The Philosophical Review. 64 (4): 527-545 (p. 532, note 9).

[19] C.E. Shannon A mathematical theory of Communication. Bell System Technical J. 1948. 27(4) pg 623-656

[20] M. Karnaugh (November). "The Map Method for Synthesis of Combinational Logic Circuits". Transactions of the American Institute of Electrical Engineers, Part I: Communication and Electronics. 1953. 72 (9): 593-599.

[21] W.V.O. Quine, Willard Van Orman. A Way to Simplify Truth Functions. The American Mathematical Monthly. 1955, 62 (9): 627-631.

[22] J. McCluskey, J. Edward. (November). "Minimization of Boolean Functions". Bell System Technical Journal. 1956, 35 (6): 1417-1444.

[23] J. Pearl, Causality: models, reasoning, and inference. Cambridge: Cambridge University Press, 2000.

[24] H. Su, N. Gorji, S. Poon, A network-based deterministic model for causal complexity. In Proceedings of the 51st Hawaii International Conference on System Sciences. http://hdl.handle.net/10125/50094. 2018

[25] A. Thiem and A. Duşa, "QCA: A package for qualitative comparative analysis," R Journal, vol. 5, pp. 87 97, 2013.

[26] S. K. Poon, K. Fan, J. Poon, C. Loy, K. Chan, X. Zhou, et al., "Analysis of herbal formulation in TCM: Infertility as a case study," in International Conference on Bioinformatics and Biomedicine Workshops, 2011, pp. 868-872.

[27] X. Zhou, J. Poon, P. Kwan, R. Zhang, Y. Wang, S. Poon, et al., "Novel two-stage analytic approach in extraction of strong herb-herb interactions in TCM clinical treatment of insomnia," 2010, pp. 258-267.

[28] S.K. Poon, A, Su, L. Chau, et al., "Causal Complexities of TCM Prescriptions; Understanding the Underlying Mechanisms of Herbal Formulation", Data Analytics for Traditional Chinese Medicine Research (Poon, J. and Poon, S.K. Ed.), Springer Publishing, 2014, pp.17-38. 